\title{
Observational results for eight long-period comets observed far from the Sun ${ }^{\star}$
}

\author{
E. Mazzotta Epifani ${ }^{1}$, D. Perna ${ }^{2}$, L. Di Fabrizio ${ }^{3}$, M. Dall'Ora ${ }^{1}$, P. Palumbo ${ }^{4}$, C. Snodgrass ${ }^{5}$, J. Licandro ${ }^{6,7}$, \\ V. Della Corte ${ }^{4}$, and G. P. Tozzi ${ }^{8}$
}

1 INAF - Osservatorio Astronomico di Capodimonte, via Moiariello 16, 80131 Napoli, Italy e-mail: epifani@oacn.inaf.it

2 LESIA, Observatoire de Paris, CNRS, UPMC, Université Paris-Diderot, 5 place Jules Janssen, 92195 Meudon, France

${ }^{3}$ Fundación Galileo Galilei - INAF, Rambla José Ana Fernández Pérez 7, 38712 Breña Baja, TF, Spain

${ }^{4}$ Universitá Parthenope, Dip. Scienze Applicate, Centro Direzionale Isola C4, 80143 Napoli, Italy

5 Max Planck Institute for Solar System Research, Max-Planck-Str. 2, 37191 Katlenburg-Lindau, Germany

${ }^{6}$ Instituto de Astrofísica de Canarias, c/vía Láctea s/n, 38200 La Laguna, Tenerife, Spain

7 Departamento de Astrofísica, Universidad de La Laguna, 38205 La Laguna, Tenerife, Spain

${ }^{8}$ INAF - Osservatorio Astrofisico di Arcetri, Largo Enrico Fermi 5, 50125 Firenze, Italy

Received 14 February 2013 / Accepted 31 October 2013

\begin{abstract}
Context. With this work we start a systematic analysis of the distant activity of several long-period comets in order to investigate the evolution of activity throughout the solar system and explore differences between comets that pass their perihelion at far or very close distances from the Sun.

Aims. We present observational data for eight long-period comets, observed for the first time beyond $r=5$ AU. Three targets have been characterised on their inward orbital branch. The others have passed their perihelion at quite large heliocentric distances $\left(r_{\mathrm{q}}\right.$ from 4.5 to $7.5 \mathrm{AU})$.

Methods. We analyse multicolour broadband images ( $V, R$, and $I$ filters) taken at the Telescopio Nazionale Galileo to characterise the dust coma of the comets and investigate their morphology, photometry, colours, and dust production.

Results. The morphological analysis shows many differences among the sample, from the large twisted structure present in the coma of comet C/2005 L3 to the regular coma envelope of C/2010 R1. The colour of the dust coma of all the comets is redder than the Sun. The Af $\rho$ value (measured in a reference aperture of radius $\left.\rho=10^{4} \mathrm{~km}\right)$ ranges from $114 \pm 2(\mathrm{C} / 2005 \mathrm{~S} 4)$ to $5091 \pm 47$ $(\mathrm{C} / 2005 \mathrm{~L} 3) \mathrm{cm}$, depicting a scenario of bodies from moderately to very active. This is confirmed by the first-order quantitative estimate of the dust mass-loss rate for the comets that was obtained from the photometric data: assuming a grain velocity of $v=20 \mathrm{~m} / \mathrm{s}$, the dust production rate is comparable with, or even significantly larger than, that measured for many short-period ("old") comets at much smaller heliocentric distances.
\end{abstract}

Key words. comets: general

\section{Introduction}

The long-period comets (LPCs) probably formed in the same region as the giant planets (the Jupiter-Neptune zone), in a warmer thermal environment than did the short-period comets (SPCs), the Kuiper belt objects (KBOs), and the Centaurs, and then scattered towards the Oort cloud region (Meech \& Svoren 2004). Moreover, recent models of the solar system (Levison et al. 2010) suggest that many other comets in the Oort Cloud may have an extrasolar origin (captures from protoplanetary discs of other stars). All the above models have strong consequences on the abundances and composition of trapped gas and on cometary activity (Meech et al. 2009).

There is much reference in the literature to "new" comets (i.e. comets that never or rarely passed through the inner solar system) being intrinsically more active at large distances from the Sun than the periodic ("old") comets (i.e. comets for which

$\star$ Based on observations collected at the Italian Telescopio Nazionale Galileo (TNG), operated on the island of La Palma by the Centro Galileo Galilei of the INAF (Istituto Nazionale di Astrofisica) at the Spanish Observatorio del Roque de los Muchachos of the Instituto de Astrofísica de Canarias. many passages inside the solar system have been recorded) (Meech 1988, 1990, 1992). As of July 2013, very few $(<20)$ LPCs have been observed and studied at distances from the Sun larger than 5-6 AU, where it is too cold for the "canonical" sublimation of crystalline water ice to explain cometary activity (Meech \& Svoren 2004).

Postperihelion activity beyond 5-6 AU could be caused by a perihelion heat wave that penetrates into volatile-rich depths in the nucleus, causing the amorphous-to-crystalline water ice transition (Notesco et al. 2003; Bar-Nun \& Laufer 2003) and the following release of hypervolatile gas. Comets that have never been close enough to the Sun to experience significant heating require a different mechanism. Up to now, only seven such comets have been observed preperihelion (Korsun \& Chorny 2003; Tozzi et al. 2003; Meech et al. 2009; Korsun et al. 2010), and five of them had then a large $(r>5 \mathrm{AU}) q$ passage.

In the recent past, the distant dust environment of the comets C/2003 O1 (LINEAR) at 7.4 AU post- $q$ (Mazzotta Epifani et al. 2009) and C/2007 D1 (LINEAR) at 9.7 AU post- $q$ (Mazzotta Epifani et al. 2010) has been quantitatively studied, deriving unexpectedly high dust production rates $\left(\sim 30 \mathrm{~kg} \mathrm{~s}^{-1}\right.$ and $>500 \mathrm{~kg} \mathrm{~s}^{-1}$, respectively), of the same order of magnitude 
Table 1. Orbital parameters of the target comets.

\begin{tabular}{lcccccccc}
\hline \hline Comet & $a^{a}[\mathrm{AU}]$ & $e^{b}$ & $q^{c}[\mathrm{AU}]$ & $t_{\mathrm{p}}{ }^{d}[\mathrm{yr}]$ & $i^{e}\left[{ }^{\circ}\right]$ & $\operatorname{Peri}^{f}\left[{ }^{\circ}\right]$ & Node $^{g}\left[{ }^{\circ}\right]$ & Classification $^{h}$ \\
\hline C/2005 L3 (McNaught) & 12158 & 0.9995 & 5.59 & 2008 Jan. 16 & 139.4 & 47.1 & 288.7 & dyn. new \\
C/2005 S4 (McNaught) & 5676 & 0.9990 & 5.85 & 2007 July 18 & 108.0 & 31.5 & 318.3 & returning \\
C/2007 JA21 (LINEAR) & 2990 & 1.0018 & 5.37 & 2006 Nov. 14 & 89.8 & 93.7 & 65.5 & dyn. new \\
C/2007 M1 (McNaught) & 1564 & 0.9948 & 7.47 & 2008 Aug. 11 & 139.7 & 52.6 & 326.8 & returning \\
C/2008 FK75 (Lemmon-Siding Spring) & 1651 & 1.003 & 4.51 & 2010 Sep. 29 & 61.2 & 80.4 & 218.3 & dyn. new \\
C/2009 S3 (Lemmon) & 3807 & 1.0017 & 6.48 & 2011 Dec. 10 & 60.4 & 129.7 & 225.1 & returning \\
C/2010 R1 (LINEAR) & 2029 & 1.0028 & 5.62 & 2012 May 19 & 156.9 & 114.5 & 343.6 & dyn. new \\
C/2011 L4 (PANSTARRS) & 1689 & 1.0002 & 0.3 & 2013 Mar. 9 & 84.0 & 333.6 & 65.7 & dyn. new \\
\hline
\end{tabular}

Notes. ${ }^{(a)}$ Semimajor axis. ${ }^{(b)}$ Orbital eccentricity. ${ }^{(c)}$ Perihelion distance. ${ }^{(d)}$ Time of perihelion passage. ${ }^{(e)}$ Inclination. ${ }^{(f)}$ J2000 argument of perihelion. ${ }^{(g)}$ J2000 longitude of ascending node. ${ }^{(h)}$ Based on the original semimajor axis in the Nakano Notes (orbital computations by Syuichi Nakano), http://www.oaa.gr.jp/ oaacs/nk.htm (the original semimajor axis is determined after the integration backward one orbit, under the influence of the Sun and planets, galactic tides, and possibly nongravitational forces and nearby stars).

Table 2. Observing circumstances.

\begin{tabular}{|c|c|c|c|c|c|c|c|c|c|}
\hline Comet & Observation time $^{a}$ (UT) & $r^{b}[\mathrm{AU}]$ & $\Delta^{c}[\mathrm{AU}]$ & $a^{d}\left[{ }^{\circ}\right]$ & $\mathrm{PA}^{e}\left[{ }^{\circ}\right]$ & $\mathrm{VV}^{f}\left[{ }^{\circ}\right]$ & $\mathrm{I} / \mathrm{O}^{g}$ & Filters $^{h}$ & Night $^{i}$ \\
\hline $\mathrm{C} / 2005 \mathrm{~L} 3$ & 2009-May-28 23:07 & 6.64 & 6.25 & 8.34 & 138.6 & 107.9 & $\mathrm{O}$ & $R[600]$ & $\mathrm{p}$ \\
\hline C/2005 S4 & 2009-May-30 01:55 & 7.52 & 7.28 & 7.63 & 188.3 & 114.9 & $\mathrm{O}$ & $V[8 \times 600], R[7 \times 600]$ & $\mathrm{p}$ \\
\hline C/2007 JA21 & 2009-May-29 01:30 & 8.47 & 7.48 & 1.61 & 178.9 & 9.3 & $\mathrm{O}$ & $V[4 \times 600], R[7 \times 600]$ & $\mathrm{p}$ \\
\hline C/2007 M1 & 2009-May-30 00:49 & 7.69 & 6.94 & 5.31 & 202.5 & 108.5 & $\mathrm{O}$ & $R[3 \times 600]$ & $\mathrm{p}$ \\
\hline C/2008 FK75 & 2011-Jun.-28 02:17 & 5.01 & 5.00 & 11.7 & 238.4 & 290.3 & $\mathrm{O}$ & $\begin{array}{c}V[8 \times 300], R[9 \times 300], \\
I[8 \times 300]\end{array}$ & $\mathrm{pp}$ \\
\hline $\mathrm{C} / 2009 \mathrm{~S} 3$ & 2011-Jun.-28 03:26 & 6.57 & 6.58 & 8.87 & 238.3 & 280.5 & I & $\begin{array}{c}V[8 \times 300], R[11 \times 300], \\
\mathrm{I}[8 \times 300]\end{array}$ & $\mathrm{pp}$ \\
\hline C/2010 R1 & 2011-Jun.-27 22:09 & 6.09 & 5.20 & 3.28 & 151.0 & 97.4 & $\mathrm{I}$ & $\begin{array}{c}V[4 \times 300], R[10 \times 300], \\
V I[4 \times 300]\end{array}$ & $\mathrm{pp}$ \\
\hline C/2011 L4 & 2011-Jun.-28 00:04 & 7.70 & 6.85 & 1.84 & 107.3 & 27.2 & I & $R[7 \times 300]$ & $\mathrm{pp}$ \\
\hline
\end{tabular}

Notes. ${ }^{(a)}$ UT time at the beginning of the imaging sequence. ${ }^{(b)}$ Heliocentric distance. ${ }^{(c)}$ Geocentric distance. ${ }^{(d)}$ Phase angle. ${ }^{(e)}$ Position angle of the extended Sun-target radius vector. ${ }^{(f)}$ Position angle of the target's heliocentric velocity vector. ${ }^{(g)}$ Orbital branch of observation; I: Inbound; O: Outbound. ${ }^{(h)}$ For each filter, the total integration time (number of images $\times$ exposure time). ${ }^{(i)}$ Quality of the night; p: photometric; pp: partly photometric.

or even larger than that of old periodic comets at much smaller heliocentric distances (see, e.g., Mazzotta Epifani \& Palumbo 2011). Unfortunately, very few similar studies for LPCs on their pre- $q$ orbital branch are presently available. Moreover the current rate of discovery of large- $q$ LPCs is quite low, at about $0.83 \mathrm{yr}^{-1}$ (Francis 2005), but new surveys such as Pan-STARRS (Kaiser et al. 2002) and the Large Synoptic Survey Telescope (LSST, Wolffs et al. 2007) will increase this rate.

In 2009 we started an observational programme for a systematic investigation of the distant dust environment of several LPCs at very large heliocentric distances, beyond the "water" zone. The comet C/2007 D1 (LINEAR) (Mazzotta Epifani et al. 2010) was observed among the sample, and its exceptional activity at almost $10 \mathrm{AU}$ post- $q$ was promptly reported.

The aim of this paper is to study the coma properties of the other eight comets in our first sample, which covers both the returning and dynamically new groups of LPCs, with some on their inbound and some on their outbound orbital branch. The study presented here increases the sample of these targets characterised by multicolour photometry when far from the Sun by $50 \%$. It adds three targets, never characterised before, to the still sparsely populated subgroup of pre- $q$ dynamically new LPCs presently characterised.

Section 2 describes observations and data reduction. In Sect. 3 we describe the observational results from each of the comets' coma characteristics (morphology and colours). Section 4 is dedicated to the discussion of the dust mass-loss rates derived from cometary photometry. Summary and conclusions are given in Sect. 5.

\section{Observations and data reduction}

The observations of eight LPCs were performed on two observing runs at the $3.52 \mathrm{~m}$ Telescopio Nazionale Galileo (TNG) at the Observatorio del Roque de los Muchachos (La Palma, Canary Islands) in May 2009 and June 2011. The target comets and their orbital parameters are listed in Table 1: three returning and five dynamically new LPCs were observed. The observing circumstances are presented in Table 2.

The images were obtained with the Device Optimised for the LOw RESolution (DOLORES) instrument ${ }^{1}$, equipped with the broadband filters $V, R$, and $I$ of the Johnson-Cousin system.

During two nights in May 2009 and one night in June 2011, several long (300 or $600 \mathrm{~s}$ ) exposures were obtained for the targets, with the telescope tracking at the nonsidereal rate corresponding to the predicted motion of the comet. Not all filters were used for all the targets, due to lack of time and faintness of the targets, see Table 2. All the images were corrected for overscan, bias, and flat-fielding in the standard manner, using tools

1 http://www.tng.iac.es/instruments/lrs/ 


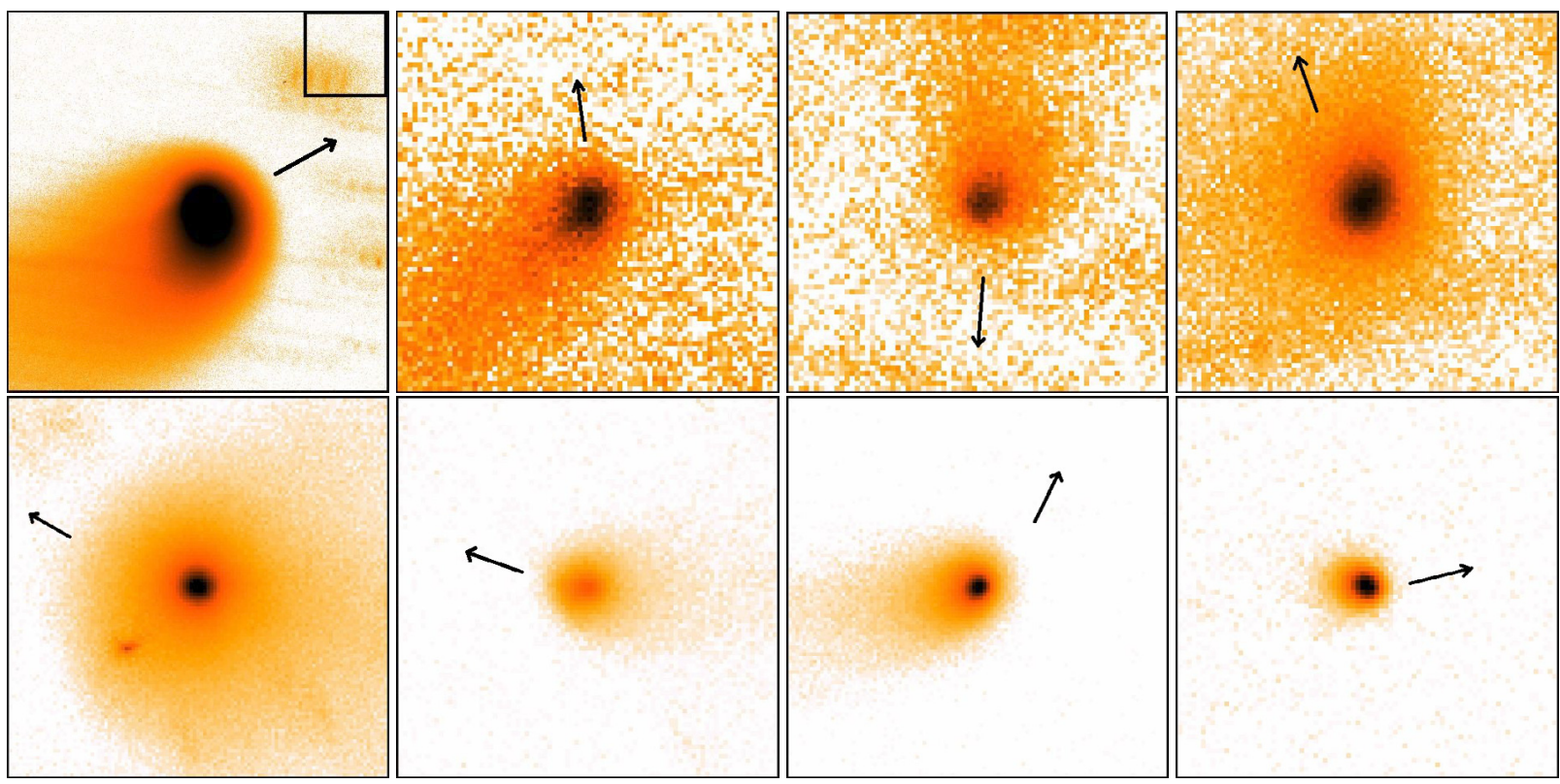

Fig. 1. From left to right and from top to bottom, median-averaged image obtained for comets C/2005 L3, C/2005S4, C/2007JA21, C/2007 M1, C/2008 FK75, C/2009 S3, C/2010 R1, and C/2011 L4 in the $R$ filter. Each image has a width of $10^{5} \mathrm{~km}$ at the comet's distance, except that for comet $\mathrm{C} / 2005 \mathrm{~L} 3$, which covers $5 \times 10^{5} \mathrm{~km}$. In this image, the black box in the upper-right corner has the same size as the other images. For all the images, $\mathrm{N}$ is up and $\mathrm{E}$ is on the left. The black arrow indicates the direction towards the Sun.

available in IRAF $^{2}$ and in ESO's Munich Image Data Analysis System (MIDAS). To perform an absolute flux calibration of the comet images, we observed appropriate fields from the list of Landolt (1992) at different airmasses each observing night. The nights in May 2009 were both photometric; the night in June 2011 was only partly photometric.

Despite the large heliocentric distance of observation, each target was easily identifiable and appeared well active in each of the single exposures. In order to increase the signal-to-noise ratio $(\mathrm{S} / \mathrm{N})$ and to remove any residual background star contribution, we median averaged the images for each target and for each filter after recentering them to the comet optocentre. We found the omet optocentre by fitting a two-dimensional Gaussian curve to the innermost pixels of the coma. Then, the sky correction was performed by subtracting a first-order polynomial sky approximation computed from the pixel areas of the same medianaveraged comet image, both containing no stars and far away from a possible coma contamination.

Figure 1 shows for each comet a subimage of the obtained median-averaged image in the $R$ filter as described above. All the images are centred on the comet optocentre. Also, $V$ and I images were obtained for some of the comets.

\section{Observational results}

\subsection{Coma morphology}

Coma morphology is a powerful tool to investigate the nucleus and coma properties by observing and interpreting different features such as bright jets, fans, and arc structures (see the recent review by Farnham 2009). The $R$ band-pass filter used $(\lambda=644 \mathrm{~nm}, F W H M 148 \mathrm{~nm})$ is the least affected by possible

\footnotetext{
2 IRAF is distributed by the National Optical Astronomical Observatory, which is operated by the Association of Universities for Research in Astronomy, Inc., under cooperative agreement with the National Science Foundation.
}

gaseous emission lines. We used images taken with this filter to search for distinct dust extended features. For this purpose we applied one or two analysis techniques to the coma images, depending on image quality and $\mathrm{S} / \mathrm{N}$.

The first technique is the radial normalisation method (Birkle \& Boehnhardt 1992), which reduces the general coma background and enhances faint coma structures. It has been applied to cometary broadband images in many previous works (e.g. Lara et al. 2009; Mazzotta Epifani \& Palumbo 2011), to which we refer for more details.

The second technique is the adaptive Laplace filtering method (Boehnhardt \& Birkle 1994), which is sensitive to gradient changes on different spatial scales, depending on the width of the spatial filter applied. We applied this method to better investigate anisotropy found by means of the radial normalisation in the dust coma of comet C/2005 L3 (see below).

Figures 2 and 3 show the processed $R$-filter images obtained for some of the target comets (those for which we obtained images with high enough $\mathrm{S} / \mathrm{N}$ ).

Comet C/2005 L3: the comet clearly shows deviation from the classical aspect of a diffuse nebulosity surrounding the nucleus. The radial-normalised image enhances the presence of a large twisted structure extending mostly in the south. A welldefined jet-like structure is evident in the Laplace-filtered image, extending in the same direction as the spiral's axis of symmetry. While the overall coma structure could be due to a fan being bent by radiation pressure into the tail direction, it could also be a hint of nucleus rotational effects in the presence of an active area on the comet's nucleus.

Comet C/2008 FK75: the comet shows some faint twisting structures that are similar to arcs and/or spirals and barely perceptible under the overall shape of the ambient coma. The presence of these multiple and successively larger nested shells could suggest a scenario of cyclic dust outflow from the nucleus, which may be linked to some specific physical nucleus properties and/or geometrical conditions. Unfortunately, snapshot 


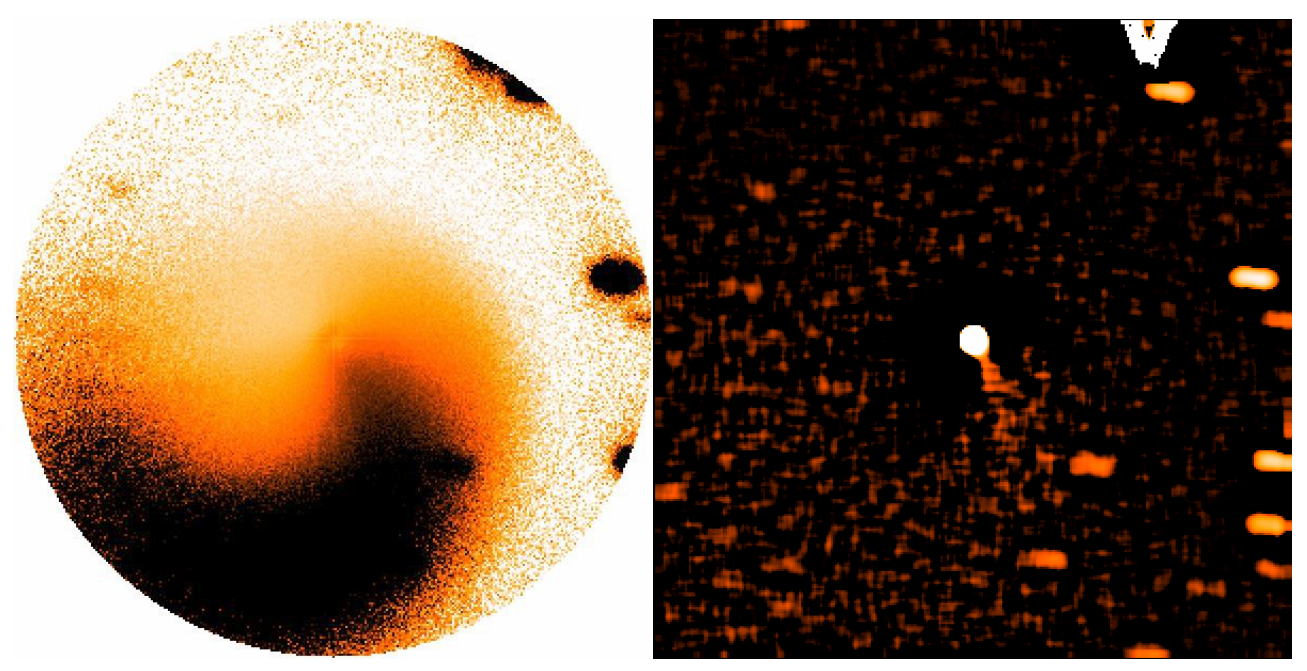

Fig. 2. Radial-normalised (left) and Laplace-filtered (right) image obtained in the $R$ filter for comet C/2005 L3. Each image is $75.9^{\prime \prime} \times 75.9^{\prime \prime}($ linear scale: $3.4 \times 10^{5} \mathrm{~km}$ ).
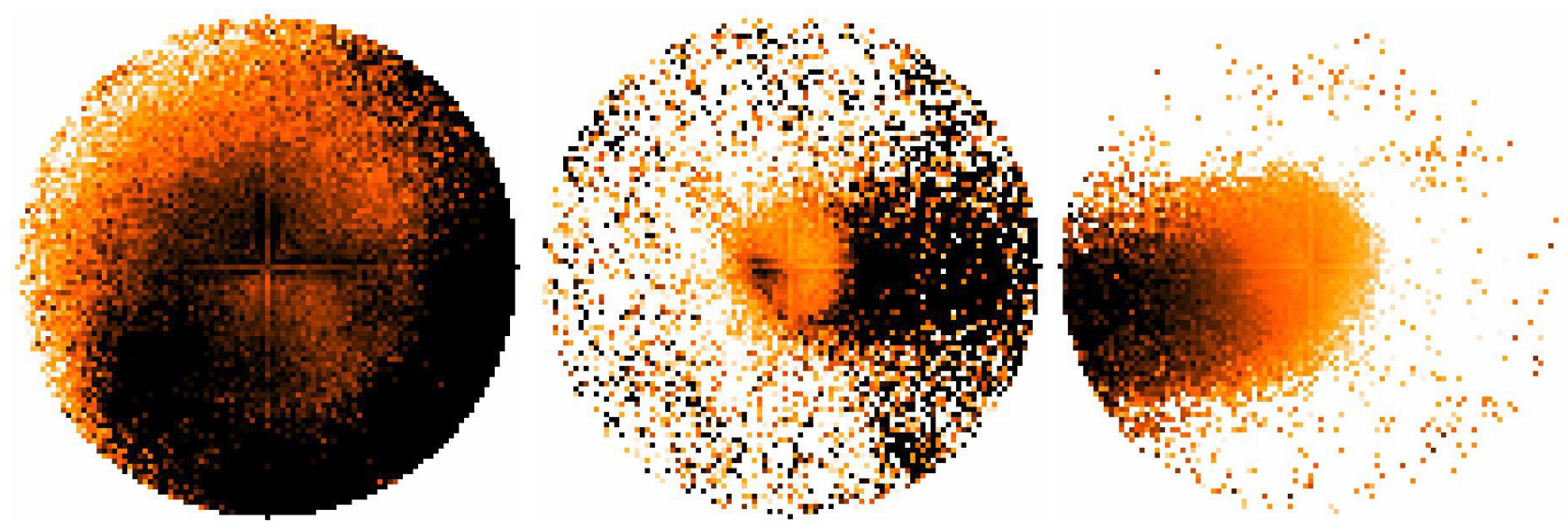

Fig. 3. From left to right, radial-normalised images obtained in the $R$ filter for comets C/2008 FK75, C/2009 S3, and C/2010 R1. Each image is $25.5^{\prime \prime} \times 25.5^{\prime \prime}$ (linear scale: $9.2 \times 10^{4} \mathrm{~km}, 1.2 \times 10^{5} \mathrm{~km}$, and $9.6 \times 10^{4} \mathrm{~km}$ for the three comets, respectively).

observations like the one presented in this paper do not allow periodicity to be enhanced and the activity throughout a rotational cycle to be charecterised. The structures are quite faint and are not clearly evident in the Laplace-filtered image.

Comet C/2009 S3: the comet is characterised by a fan-shaped tail extended along the $\mathrm{W}$ direction. In addition, an excess in the anti-tail direction is well visible above the surrounding "regular" coma in the radial-normalised image. We checked whether this excess could be due to an artifact of the image processing, but it is present in the same position relative to the comet in all the single images used for the final median average. As the comet optocentre moves about 68 arcsec in the field between the first and the last image of the sequence, we can exclude the possibility that the excess is due to a residual field star and interpret it as an asymmetric dust outflow that is strongly limited in space.

Comet $C / 2010 \mathrm{Rl}$ : this comet acts as an example of a regular coma envelope, with no evidence of subtle radial or azimuthal structure present in the dust coma.

For comets C/2005 S4, C/2007 JA21, C/2007 M1, and $\mathrm{C} / 2011 \mathrm{~L} 4$, the obtained $\mathrm{S} / \mathrm{N}$ was not high enough to get reliable results from the image processing. The general aspect of these comets is quite standard and similar in all the filters in which we obtained images (see Fig. 1): a small condensation close to the nucleus and a more or less extended broad tail-like structure. For comet C/2011 L4, the tail-like elongation was still very small at the time of observation.

\subsection{Coma photometry}

The integrated magnitudes of the coma, which are derived for all the filters in the reference optical aperture of $\rho=10^{4} \mathrm{~km}$ centred on the optocentre, are listed in Table 3. The final uncertainty in the magnitude comes from the calibration error $\sigma_{\text {calib }}$ (rms of the standard stars fit, weighted by the star counts against the background).

Table 3 also summarises the coma colours obtained in the reference optical aperture. From the comparison of cometary values with the solar ones, we observe that most target comets appear from slightly to significantly redder than the Sun, even if for some of them the error on the colour is too large to secure a significant reddening trend. However, this comparison should be carefully regarded since the colour index strictly depends on observational and data reduction constraints (e.g. aperture size used to compute the magnitude). Moreover, coma colours 
Table 3. Observational results for the target comets.

\begin{tabular}{lccccc}
\hline \hline Comet & \multicolumn{3}{c}{ Integrated magnitude $^{a}$} & \multicolumn{2}{c}{ Colour index $^{b}$} \\
& $V$ & $R$ & $I$ & $(V-R)$ & $(R-I)$ \\
\hline C/2005 L3 & - & $16.58 \pm 0.02$ & - & - & - \\
C/2005 S4 & $21.68 \pm 0.02$ & $21.26 \pm 0.02$ & - & $0.42 \pm 0.03$ & - \\
C/2007 JA21 & $21.59 \pm 0.01$ & $21.20 \pm 0.02$ & - & $0.39 \pm 0.02$ & - \\
C/2007 M1 & - & $19.72 \pm 0.02$ & - & - & - \\
C/2008 FK75 & $17.9 \pm 0.1$ & $17.39 \pm 0.09$ & $17.09 \pm 0.12$ & $0.51 \pm 0.13$ & $0.30 \pm 0.15$ \\
C/2009 S3 & $20.0 \pm 0.1$ & $19.51 \pm 0.09$ & $19.04 \pm 0.12$ & $0.49 \pm 0.13$ & $0.47 \pm 0.15$ \\
C/2010 R1 & $18.7 \pm 0.1$ & $18.23 \pm 0.09$ & $17.86 \pm 0.11$ & $0.44 \pm 0.13$ & $0.44 \pm 0.15$ \\
C/2011 L4 & - & $19.03 \pm 0.09$ & - & - & - \\
\hline Solar & \multicolumn{7}{c}{} \\
\hline
\end{tabular}

Notes. ${ }^{(a)}$ Integrated coma magnitude derived for all the filters in the reference optical aperture of $\rho=10^{4} \mathrm{~km}$, centred on the optocentre. The errors derive from the photometric calibration. ${ }^{(b)}$ Colour indexes integrated in the reference optical aperture of $\rho=10^{4} \mathrm{~km}$, centred on the optocentre. The solar colours are those in the modern Johnson-Cousins system (Holmberg et al. 2006).

are integrated properties and, as such, depend significantly on short-term scale variations in the spatial distribution of the dust.

The colour changes with radial distance from coma center are interesting to explore in order to investigate the whole dust environment of the coma, e.g. if optical properties of dust grains vary within the coma due to processes such as sublimation or fragmentation. For most of the comets $(V-R$ for five comets, $R-I$ for three), our data allow a first-order investigation of the colour trend with cometocentric radial distance. Actually, the error on the colour indices is sometimes (i.e. for some comets in the sample) too large to secure a significant trend with the cometocentric distance. Moreover, the apparent projected size of the coma is generally different from comet to comet, thus preventing a comparative analysis of the indices behaviour among the sample. Nevertheless, when considering the actual values of colour indices, we noticed that for comets C/2007 JA21, C/2005 S4, $\mathrm{C} / 2009 \mathrm{~S} 3$, and $\mathrm{C} / 2010 \mathrm{R} 1$ the trend ranges from being constant $(\mathrm{C} / 2009 \mathrm{~S} 3, \mathrm{C} / 2010 \mathrm{R} 1)$ to an increasing reddish colour with the radial distance (C/2007 JA21, C/2005 S4). The only remarkable exception is comet $\mathrm{C} / 2008 \mathrm{FK} 75$, for which $(V-R)$ and mostly $(R-I)$ colours decrease with the radial distance, indicating that there are significant variations towards bluer dust colour with increasing radial distance. This could indicate sublimation of ices present on dust grains, as already observed in, for example, comet 103P/Hartley 2 (Lara et al. 2011).

\section{3. $\operatorname{Af} \rho$}

The A $f \rho$ value expressed in cm (A'Hearn et al. 1984), where $A$ is the average grain geometric albedo, $f$ the filling factor in the aperture field of view, and $\rho$ the linear radius of the aperture at the comet, i.e. the sky-plane radius, is usually used as a proxy for the cometary dust production. When the cometary coma is in a steady state, A $f \rho$ is an aperture-independent parameter.

Figure 4 shows the trend of $R$-band A $f \rho$ for the target comets with the cometocentric distance (computed up to a distance for which the coma is measured to be $3 \sigma$ above the background: this also allows an indication of the projected extension of the dust coma of each target to be obtained). After a more or less steep increase in the inner part of the coma, which is affected by the seeing and observable in all targets, for most of the comets (C/2005 L3, C/2007 M1, C/2008 FK75 C/2009 S3, $\mathrm{C} / 2010 \mathrm{R} 1$, and $\mathrm{C} / 2011 \mathrm{~L} 4)$ it is possible to clearly identify a decrease in Af $\rho$ with the nucleocentric distance $\rho$. A similar decrease has been often attributed to non steady state dust emission and possibly dust grain fading or destruction (Lara et al. 2004; Tozzi et al. 2003) and/or to fresh dust production within the coma, even if the latter process is more likely to be observed during close passages to the Sun (Tozzi et al. 2011). This decrease is not observed for the remaining two comets (C/2005 S4 and C/2007 JA21), but it could be that the relatively small apparent coma extension and low activity level prevented such a detection with our data. The decrease in A $f \rho$ is clearly observable, with a similar relatively small apparent coma extension in comets C/2010 R1 and C/2011 L4.

The Af $\rho$ values for the target comets, which are derived for the $R$ filter in the reference optical aperture of $\rho=10^{4} \mathrm{~km}$, are listed in Table 4, together with the maximum $R$-band A $f \rho$ and the relative aperture radius. The $\mathrm{A} f \rho$ trend is quite different among the sample. For comets C/2010 R1 and C/2011 L4, for example, the maximum is reached at low $\rho$ and followed by a quite steep decrease, while for comets C/2005 S4 and C/2007 JA21 (of similar apparent coma extension) the maximum is reached later. Then the trend is quite constant with the cometocentric distance.

It is hard to choose one A $f \rho$ value for each comet to compare results among our sample and with values in literature. In fact, A $f \rho$ is often used to compare the dust production rate between comets, but one should keep in mind that it involves several observational and physical parameters: the aperture size if the A $f \rho$ is not constant with the cometocentric distance; the geometric albedo of the grains, which depends in turn on the observing phase angle and wavelength; the grain size distribution. Nevertheless, as a general rule it is assumed that large A $f \rho$ values indicate high dust activity. The results obtained for the eight targets in our sample confirm indeed that these comets are moderately to very active objects. Figure 5 shows the comparison of $R$-band Af $\rho$ obtained in the reference aperture of $\rho=10^{4} \mathrm{~km}$ in the present paper with the values obtained for several cometary objects (SPCs and LPCs) in the past, from both snapshot and monitoring observations.

Comet C/2005 L3 appears to be the most active comet in our sample, but all the comets show $R$-band A $f \rho$ values at least of the same order of, but in many cases much greater than, those measured for other active objects (also including different dynamical groups) at much smaller heliocentric distances, where Af $\rho$ is supposed to be larger. In particular, Fig. 5 (right-hand panel) shows that the median value of LPCs' A $f \rho$, both close to $(<2-3 \mathrm{AU})$ and far from ( $>3 \mathrm{AU})$ the Sun, is larger than values measured for SPCs (the direct comparison is obviously impossible above $5 \mathrm{AU})$. 

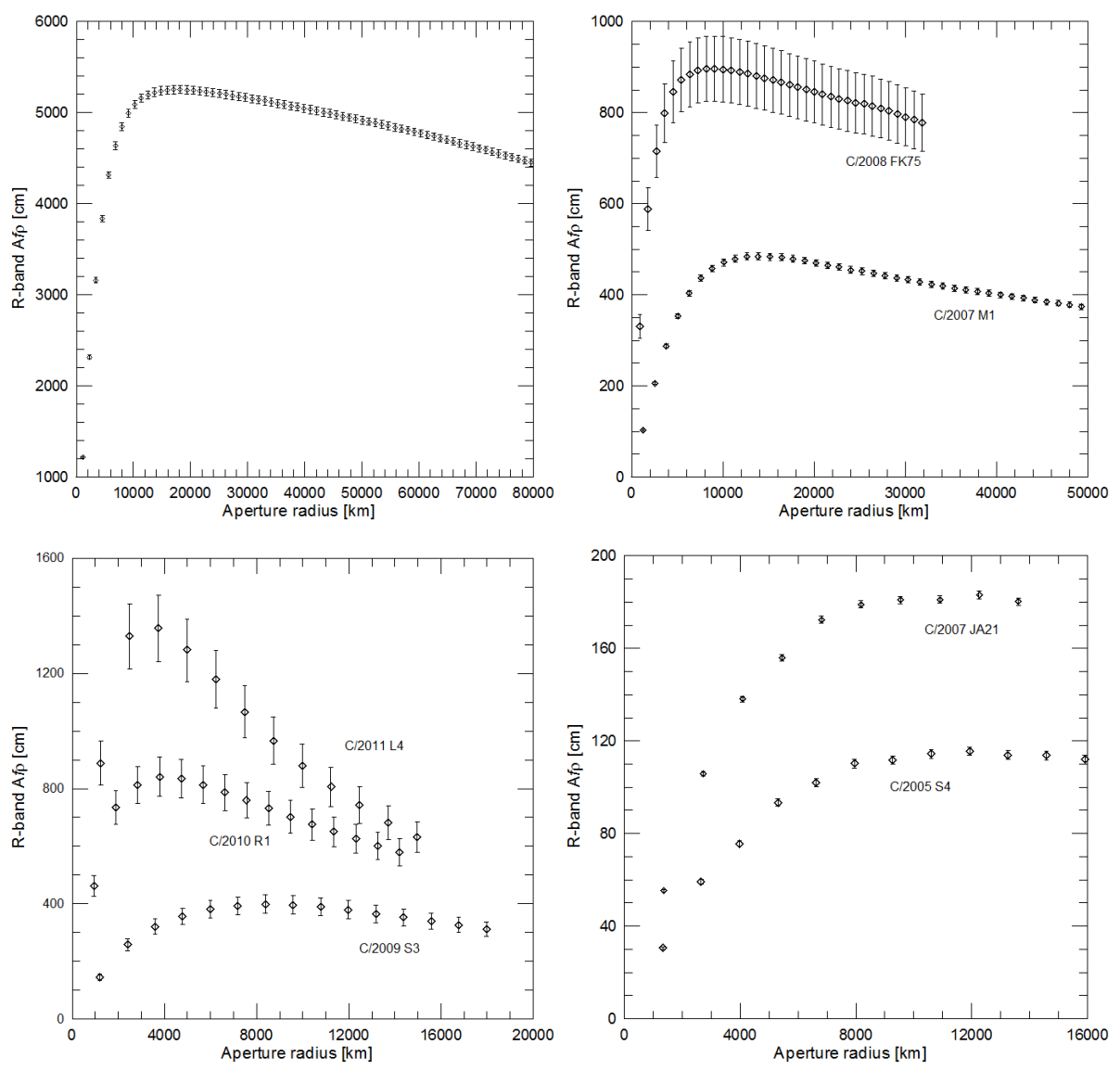

Fig. 4. $R$-band Af $\rho$ trend versus cometocentric distance measured for target comets. Top left: comet C/2005 L3; top right: comets C/2007 M1 and C/2008 FK75; bottom left: comets C/2009 S3, C/2010 R1 and C/2001 L4; bottom right: comets C/2005 S4 and C/2007 JA21.

Table 4. Af $\rho$ values for the target comets, derived for all the filters in the reference optical aperture of $\rho=10^{4} \mathrm{~km}$ and centred on the optocentre.

\begin{tabular}{lccc}
\hline \hline Comet & $\begin{array}{c}\mathrm{A} f \rho[\mathrm{cm}] \\
{\left[\rho=10^{4} \mathrm{~km}\right]}\end{array}$ & $\mathrm{A} f \rho_{\max }$ & $\rho_{\max }[\mathrm{km}]$ \\
\hline C/2005 L3 & $5091 \pm 47$ & $5255 \pm 46$ & 18204 \\
$\mathrm{C} / 2005$ S4 & $114 \pm 2$ & $116 \pm 2$ & 11927 \\
$\mathrm{C} / 2007 \mathrm{JA} 21$ & $181 \pm 2$ & $183 \pm 2$ & 12255 \\
$\mathrm{C} / 2007 \mathrm{M} 1$ & $471 \pm 7$ & $484 \pm 8$ & 12633 \\
$\mathrm{C} / 2008$ FK75 & $895 \pm 72$ & $896 \pm 72$ & 9102 \\
$\mathrm{C} / 2009$ S3 & $396 \pm 32$ & $398 \pm 32$ & 8385 \\
$\mathrm{C} / 2010 \mathrm{R} 1$ & $703 \pm 56$ & $842 \pm 68$ & 3786 \\
$\mathrm{C} / 2011 \mathrm{~L} 4$ & $880 \pm 75$ & $1357 \pm 115$ & 3742 \\
\hline
\end{tabular}

\section{Dust mass production rate}

The Af $\rho$ parameter is considered a good proxy for the dust activity, but it does not directly provide the dust production rate $Q_{\mathrm{d}}$. To obtain a first-order quantitative estimate of the dust mass-loss rate for our target comets, we applied a "photometric model", which relates the optical photometry to the dust production rate via some realistic assumptions on the cometary dust environment. The method is derived from the one used by Jewitt (2009) to compute the dust production rate of active Centaurs in the region between 5 and $12 \mathrm{AU}$; it was adapted and already successfully applied by our group to cometary environment. We refer to
Mazzotta Epifani et al. (2010) and Mazzotta Epifani \& Palumbo (2011) for further details on the method. Here we recall that the method consists of estimating the total cross section $C_{\mathrm{d}}$ of the coma dust particles in a conveniently chosen annulus by means of coma photometry, following the expression by Russell (1916): $p_{\mathrm{R}} \Phi(\alpha) C_{\mathrm{d}}=2.25 \times 10^{22} \pi r^{2} \Delta^{2} 10^{-0.4\left(m_{\mathrm{d}}-m_{\mathrm{S}}\right)}$ ( $r$ and $\Delta$ : heliocentric and geocentric distances, $\alpha$ : phase angle, $m_{S}$ : magnitude of the Sun in the $R$ filter; $m_{\mathrm{d}}$ : apparent $R$ magnitude in the annulus; $p_{\mathrm{R}}$ : geometric albedo). The annulus is selected in the coma such that any possible nucleus contribution is excluded and the effects of residual sky background are minimised. The dust cross section $C_{\mathrm{d}}$ derived from the photometry is then related to the dust mass $M_{\mathrm{d}}$ through the adoption of a power-law dust size distribution. Finally, the dust mass-loss rate $Q_{\mathrm{d}}$ is obtained by considering the time of residence of dust grains in the projected coma annulus, which is dependent on the radial outflow speed of the dust grains at the heliocentric distance $r$ of observation.

Several realistic assumptions are needed to derive quantitative values of $Q_{\mathrm{d}}$ :

Geometric albedo $p_{\mathrm{R}}$ : we adopted the typical value for comet nuclei of $p_{\mathrm{R}}=0.04$; this is an important factor in the uncertainty in our calculations since the actual albedo of dust grains is unknown.

Phase darkening $\Phi(\alpha)$ : the shape of this function depends upon a variety of properties of dust particles, including the distribution in size, roughness, and albedo. We adopted "Schleicher's method" (Schleicher et al. 1998; Schleicher 2007), which reckons on the shallower dust comae phase function with respect to that of the bare cometary nucleus. Since all the comets 

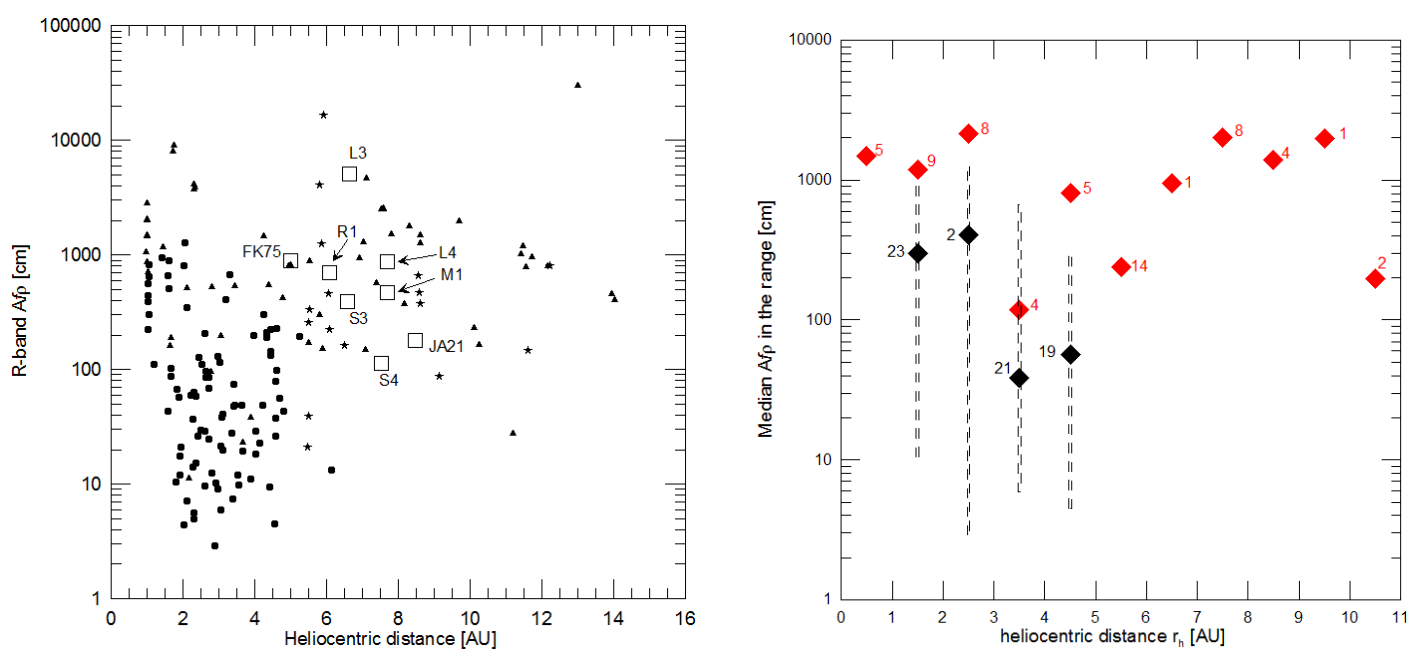

Fig. 5. Left: $R$-band A $f \rho$ values measured for target comets (open squares) compared to values derived for several minor bodies: SPCs (dots), active Centaurs (stars), and LPCs (triangles). Literature was searched for both snapshot and monitoring observations: Mazzotta Epifani \& Palumbo (2011, and references therein), Mazzotta Epifani et al. (2008, and references therein), Solontoi et al. (2012), Szabó et al. (2002), Ivanova et al. (2011), Jewitt (2009), Mazzotta Epifani et al. (2011, 2006), Bauer et al. (2003), Lara et al. (2003), Mazzotta Epifani et al. (2010), Korsun et al. (2010), Mazzotta Epifani et al. (2009, and references therein), Meech et al. (2009). (Right) Median R-band Af $\rho$ values in heliocentric distance ranges of $1 \mathrm{AU}$ for SPCs (black diamonds) and LPCs (red diamonds). The number of values available to compute the median is indicated close to each symbol. The black vertical dashed lines indicate the "cloud" (min-max values) for each point in the SPCs' sample.

in our sample were observed at small $\left(<12^{\circ}\right)$ phase angles, we used the tabulated values of Schleicher's curve normalised at $0^{\circ}$ (asteroid. lowell.edu/comet/dustphaseHM_table.txt).

Power-law dust size distribution: numerical modelling of dust tails for some LPCs (Fulle et al. 1998; Korsun \& Chorny 2003; Mazzotta Epifani et al. 2009; Korsun et al. 2010) suggests the adoption of the plausible value of $q=-3.5$ for grains between $a_{-}=10 \mu \mathrm{m}$ and $a_{+}=1 \mathrm{~cm}$ for compact cometary grains of bulk density $\rho=1000 \mathrm{~kg} / \mathrm{m}^{3}$. This is similar to the dust environment assumed for several SPC tails (see a summary in Fulle 1999) and for active Centaurs (Jewitt 2009).

Dust outflow velocity $v(r)$ : in the past, the application of an inverse numerical model to coma images allowed reconstruction of the dynamical evolution of the dust environment for a number of comets, including the dust grain velocity (strictly dependent on dust size), at large heliocentric distance (e.g. Mazzotta Epifani et al. 2009; Korsun \& Chorny 2003). In this work, we considered a dust grain velocity between 1 and an estimated realistic upper limit of $50 \mathrm{~m} / \mathrm{s}$.

Table 5 summarises, for each comet, the values derived for all the model quantities and the dust production rate $Q_{\mathrm{d}}$ for the reference velocity value of $v=20 \mathrm{~m} / \mathrm{s}$.

Figure 6 shows the dust mass-loss rate for the target comets, compared with values found in literature for other minor bodies: SPCs, active Centaurs, and other LPCs. A direct comparison between $Q_{\mathrm{d}}$ is not so straightforward since several model and observational factors highly influence the resulting dust mass-loss rate. For example, our results, given in terms of a lower-to-upper limit, are based on a realistic assumption of the grain velocity, which remains unknown at present (together with its dependence on grain size). Furthermore, there is a surprising lack of dust production rate measurements for LPCs at short heliocentric distances.

Nevertheless, from the general trend pictured in Figs. 5 and 6 it is possible to conclude that it is not an observational bias that LPCs are more active than SPCs. It is true that the more distant LPCs would not have been discovered if they were of low activity (whereas nearby short comets can be), and it is also true that
Table 5. Model dust mass-loss rate for target comets.

\begin{tabular}{lcccc}
\hline \hline Comet & $m_{\mathrm{d}}{ }^{a}$ & $\begin{array}{c}C_{\mathrm{d}}{ }^{b} \\
{\left[\mathrm{~m}^{2}\right]}\end{array}$ & $\begin{array}{c}M_{\mathrm{d}}{ }^{c} \\
{[\mathrm{~kg}]}\end{array}$ & $\begin{array}{c}Q_{\mathrm{d}}{ }^{d} \\
{[\mathrm{~kg} / \mathrm{s}]}\end{array}$ \\
\hline C/2005 L3 & 17.25 & $7.59 \times 10^{9}$ & $3.20 \times 10^{8}$ & 1407 \\
C/2005 S4 & 21.83 & $1.94 \times 10^{8}$ & $8.20 \times 10^{6}$ & 31 \\
C/2007 JA21 & 21.97 & $2.42 \times 10^{8}$ & $1.02 \times 10^{7}$ & 38 \\
C/2007 M1 & 20.23 & $7.22 \times 10^{8}$ & $3.04 \times 10^{7}$ & 121 \\
C/2008 FK75 & 18.39 & $1.11 \times 10^{9}$ & $4.68 \times 10^{7}$ & 257 \\
C/2009 S3 & 20.16 & $5.83 \times 10^{8}$ & $2.46 \times 10^{7}$ & 103 \\
C/2010 R1 & 19.26 & $6.75 \times 10^{8}$ & $2.42 \times 10^{7}$ & 128 \\
C/2011 L4 & 20.45 & $5.16 \times 10^{8}$ & $2.17 \times 10^{7}$ & 87 \\
\hline
\end{tabular}

Notes. ${ }^{(a)}$ Coma magnitude in the projected annulus between $\phi=1^{\prime \prime}$ and $\phi^{\prime}=2^{\prime \prime}$ (see text). ${ }^{(b)}$ Derived dust cross section. ${ }^{(c)}$ Derived dust mass in the coma annulus. ${ }^{(d)}$ Derived dust mass-loss rate for grains crossing the projected coma annulus at the reference velocity value of $v=20 \mathrm{~m} / \mathrm{s}$.

very distant cometary activity (i.e. $>5$ AU) can never be compared for the two groups. But when comparing activity close to the Sun, which is mainly driven by water, the average activity of LPCs is greater than that of SPCs. One potential explanation is the fact that SPCs are much older (having spent much more time close to the Sun than LPCs) and therefore have dust mantles and/or are depleted in volatiles.

\section{Summary and conclusions}

We present multicolour broad band photometry of a sample of eight long-period comets, observed for the first time at heliocentric distance $r>5$ AU with the $3.52 \mathrm{~m}$ TNG telescope. Three of them are long-period, returning comets, and five are dynamically new comets. Some of the target comets were observed on their inbound orbital branch, while the others were observed after 


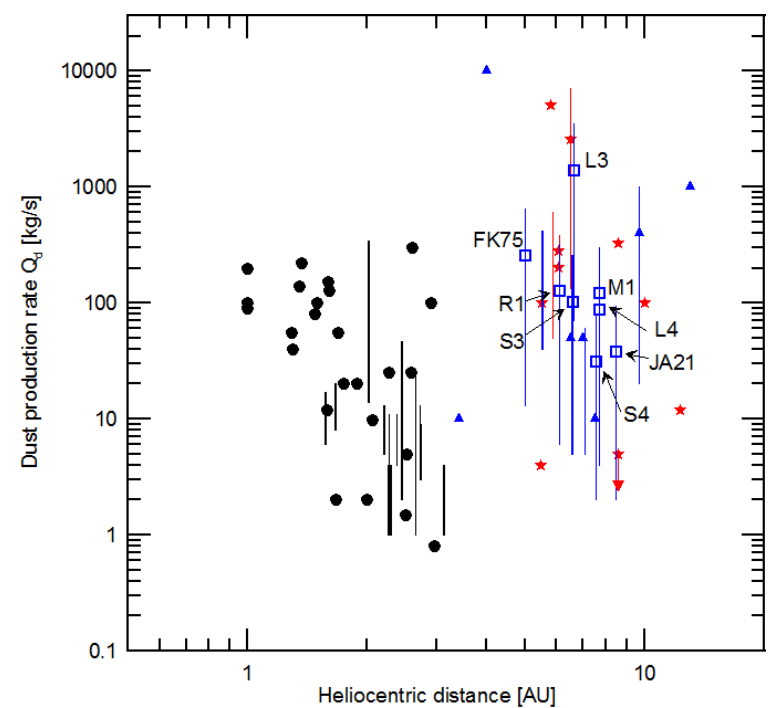

Fig. 6. Dust mass-loss rate of target comets (blue open squares: values computed for the reference velocity of $v=20 \mathrm{~m} / \mathrm{s}$; blue vertical lines: min-max range computed by means of the photometric model for grain velocity from 1 to $50 \mathrm{~m} / \mathrm{s}$ ) compared to values for several minor bodies: SPCs (black dots: single observations; black vertical lines: min-max range derived for typical/depleted comet classification or range computed by means of the photometric model for different grain velocity values; see Mazzotta Epifani \& Palumbo (2011; their Fig. 12) and references therein), active Centaurs (red stars: single observations; red vertical lines: min-max range computed by means of the photometric model for different grain velocity values; see Mazzotta Epifani et al. 2006, 2011; Bauer et al. 2003; Ivanova et al. 2011), and LPCs (blue triangles: single observations; blue vertical lines: min-max range computed by means of the photometric model for different grain velocity values; see Fulle et al. 1998; Mazzotta Epifani et al. 2009; Ivanova et al. 2011).

their perihelion passage at heliocentric distance $r_{q}>5.6 \mathrm{AU}$, i.e. well outside the so-called "water zone".

Our main results can be summarised as follows:

1. Morphological analysis was performed for some of the target comets: they vary considerably in appearance. Comet C/2005 L3 (McNaught) shows a large twisted structure. Comet C/2008 FK75 (Lemmon-Siding Spring) shows some faint twisting structures, similar to arcs and/or spirals and barely perceptible under the overall shape of the ambient coma. Comet C/2009 S3 (Lemmon) is characterised by a fan-shaped tail, with an excess in the anti-tail direction that is interpreted as an asymmetric dust outflow that is strongly limited in space.

2. The analysis of multiband photometry data showed that most target comets are slightly to significantly redder than the Sun, even if for some of them the error on the colour is too large to secure a significant reddening trend, e.g. with the radial cometocentric distance. Comet C/2008 FK75 shows indications of colour indices (mostly $(R-I))$ decreasing with the radial distance, indicating that there are significant variations towards bluer dust colour with increasing radial distance. This could point to sublimation of ices present on dust grains.

3. The $R$-band Af $\rho$ values in the reference aperture of $\rho=10^{4} \mathrm{~km}$ range from $114 \pm 2 \mathrm{~cm}$ for comet $\mathrm{C} / 2005$ S4 (McNaught) to the extraordinarily high value of $5091 \pm$ $47 \mathrm{~cm}$ for comet C/2005 L3 (McNaught).

4. By means of a "photometric model", which relates the optical photometry to the dust production rate, we obtained a first-order quantitative estimate of the dust mass-loss rate for the target comets. Assuming an average grain radius in the coma of $\langle a\rangle=30 \mu \mathrm{m}$ and a reference grain outflow velocity of $v=20 \mathrm{~m} / \mathrm{s}$, a dust mass-loss rate $\frac{\mathrm{d} M_{\mathrm{d}}}{\mathrm{d} t}$ from $31(\mathrm{C} / 2005 \mathrm{~S} 4$ (McNaught)) to $1.4 \times 10^{3}$ (C/2005 L3 (McNaught)) kg/s was derived among the sample.

All the results obtained during the observational campaign and summarised above confirm the scenario of "new" comets being intrinsically more active than periodic "old" comets. After their passage at a quite distant perihelion, five comets were observed on their outward orbital branch. Their post- $q$ activity is variable among the sample: e.g. C/2005 L3 (McNaught) and C/2005 S4 (McNaught) both passed at $q \approx 6 \mathrm{AU}$ in their present passage, and their dust mass-loss rate values, measured at similar heliocentric distances, bracket the values obtained for all the targets. This confirms the extreme variability that can be found inside a sample of targets traditionally seen as a "group", and probably reflects the different size, size evolution (e.g. fragmentation), volatile compositions, and, in turn, the different formation regions of these bodies. Moreover, these comets (especially the dynamically new ones) have never or very rarely been close to the Sun enough to experience a significant heating that would explain, at least partially, the post- $q$ release of hypervolatile gas at large heliocentric distances. These results pose important constraints on the studies on the mechanism(s) required to model their activity.

Another interesting issue explored by this paper, which opens many future observational perspectives, is the analysis of very distant, pre- $q$ cometary activity for three long-period comets never characterised before. Two of them are dynamically new in our solar system: C/2010 R1 (LINEAR) passed its perihelion at 5.6 AU in May 2012 and C/2011 L4 (PANSTARRS) did so at 0.3 AU in March 2013. They will offer in the near future the possibility to follow up and study the evolution along their orbit of two Oort cloud comets and to explore the differences of two (maybe) similar bodies, each with a very different destiny inside our solar system.

Acknowledgements. We gratefully acknowledge funding from Italian Space Agency (ASI) under contract I/062/08/00. J.L. gratefully acknowledges support from the Spanish "Ministerio de Ciencia e Innovación" project AYA2011-29489C03-02. C.S. received funding from the European Union Seventh Framework Program (FP7/2007-2013) under grant agreement No. 268421.

\section{References}

A'Hearn, M. F., Schleicher, D. G., Feldman, P. D., Millis, R. L., \& Thompson, D. T. 1984, AJ, 89, 579

Bar-Nun, A., \& Laufer, D. 2003, Icarus, 161, 157

Bauer, J. M., Fernandez, Y. R., \& Meech, K. J. 2003, PASP, 115, 981

Birkle, K., \& Boehnhardt, H. 1992, Earth, Moon, and Planets, 57, 191

Boehnhardt, H., \& Birkle, K. 1994, A\&AS, 107, 101

Farnham, T. L. 2009, PSS, 57, 1192

Francis, P. J. 2005, AJ, 635, 1348

Fulle, M. 1999, Planet. Space Sci., 47, 827

Fulle, M., Cremonese, G., \& Bóhm, C. 1998, AJ, 116, 1470

Holmberg, J., Flynn, C., \& Portinari, L. 2006, MNRAS, 367, 449

Ivanova, O. V., Skorov, Y. V., Korsun, P. P., Afanasiev, V. L., \& Blum, J. 2011, Icarus, 211, 559

Jewitt, D. C. 2009, AJ, 137, 4296

Kaiser, N., Aussel, H., Burke, B. E., et al. 2002, Proc. SPIE, 4836, 154

Korsun, P. P., \& Chorny, G. F. 2003, A\&A, 410, 1029

Korsun, P. P., Kulyk, I. V., Ivanova, O. V., et al. 2010, Icarus, 210, 916

Landolt, A. U. 1992, AJ, 104, 1, 340

Lara, L. M., Licandro, J., \& Tozzi, G. P. 2003, A\&A, 404, 373

Lara, L. M., Tozzi, G. P., Boehnhardt, H., DiMartino, M., \& Schulz, R. 2004, A\&A, 422, 717

Lara, L. M., Licandro, J., \& Tozzi, G. P. 2009, A\&A, 497, 843 
E. Mazzotta Epifani et al.: The distant activity of eight long-period comets

Lara, L. M., Lin, Z.-Y., \& Meech, K. 2011, A\&A, 532, A87

Levison, H. F., Duncan, M. J., Brasser, R., \& Kaufmann, D. E. 2010, Science, 329,187

Mazzotta Epifani, E., \& Palumbo, P. 2011, A\&A, 525, A62

Mazzotta Epifani, E., Palumbo, P., Capria, M. T., et al. 2006, A\&A, 460, 935

Mazzotta Epifani, E., Palumbo, P., Capria, M. T., et al. 2008, MNRAS, 390, 265

Mazzotta Epifani, E., Palumbo, P., Capria, M. T., et al. 2009, A\&A 502, 355

Mazzotta Epifani, E., Dall'Ora, M., Di Fabrizio, L., et al. 2010, A\&A, 513, A33

Mazzotta Epifani, E., Dall'Ora, M., Perna, D., Palumbo, P., \& Colangeli, L. 2011, MNRAS, 415, 3097

Meech, K. J. 1988, BAAS, 20, 835

Meech, K. J. 1990, BAAS, 22, 1103

Meech, K. J. 1992, BAAS, 24, 993
Meech, K. J., \& Svoren, J. 2004, in Comets II, eds. M. Festou, H. U. Keller, \& H. A. Weaver (Tucson: Univ. Arizona Press)

Meech, K. J., Pittichova, J., Bar-Nun, A., et al. 2009, Icarus, 201, 719

Notesco, G. A., Bar-Nun, A., \& Owen, T. 2003, Icarus, 162, 183

Russell, H. N. 1916, ApJ, 43, 173

Schleicher, G. 2007, Icarus, 190, 406

Schleicher, D. G., Millis, R. L., \& Birch, P. V. 1998, Icarus, 132, 397

Solontoi, M., Ivezic, Z., Juric, M., et al. 2012, Icarus, 218, 571

Szabó, G. M., Kiss, L. L., Sárneczky, K., \& Sziládi, K. 2002, A\&A, 384, 702

Tozzi, G. P., Boehnhardt, H., \& LoCurto, G. 2003, A\&A, 398, L41

Tozzi, G. P., Patriarchi, P., Boehnhardt, H., et al. 2011, A\&A, 531, A54

Wolffs, S., Sweeney, D., Tyson, J. A. et al. 2007, AAS meeting \#211, BAAS, 39, 978 